\title{
Tensiones entre el emprendimiento y el trabajo autónomo en las políticas europeas de empleo: un nuevo capítulo en la relación entre el "trabajo como empleo" y el "trabajo sobre sí"
}

\author{
Laureano Martínez Sordoni ${ }^{1}$; Patricia Amigot Leache ${ }^{2}$
}

Recibido: 07 de septiembre de 2017 / Aceptado: 21 de febrero de 2018

Resumen. Este artículo aborda las medidas sobre impulso del emprendimiento promovidas por las instituciones europeas y adoptadas en España en los últimos años. En particular, el artículo problematiza el vínculo entre "emprendimiento" y "empleo autónomo" que caracteriza al diseño de estas políticas. A partir de un análisis de fuentes documentales, se pone de relieve que la vinculación entre emprendimiento y empleo autónomo da lugar a dos procesos simultáneos: por un lado, una contradicción entre sus objetivos -la creación de empleo, el crecimiento económico y la innovación-y los instrumentos propuestos para cumplirlos. Por otro, la promoción de un modelo cultural que se construye en oposición a la idea de "empleo asalariado" y que desplaza los riesgos sociales hacia el individuo, dando lugar a una ruptura de los modelos solidaristas de políticas sociales y de empleo.

Palabras Clave: emprendimiento; empleo autónomo; política social europea; análisis discursivo de políticas públicas.

\section{[en] Tensions between entrepreneurship and self-employment in European policies: a new chapter in the relationships between "work as employment" and "work on oneself"}

\begin{abstract}
This article deals with entrepreneurship policies fostered by European institutions and adopted in Spain in recent years. In particular, it reflects on the link between "entrepreneurship" and "self-employment" that characterizes the design of these measures. Based on an analysis of documentary sources, the article highlights that the link between entrepreneurship and self-employment leads to two simultaneous processes: on the one hand, a contradiction between its objectives -job creation, economic growth and innovation- and the instruments proposed to reach them. On the other hand, the promotion of a cultural model that is constructed in opposition to the idea of "wage employment" and that shifts social risks towards the individual leading to the delegitimization of solidarity models of social and employment policies.
\end{abstract}

Keywords: entrepreneurship, self-employment, European social policy, discursive approach to public policy.

Sumario: 1. Introducción. 2. Las tensiones entre el autoempleo y la creación de empresas. 2.1. Problemas en torno a la amplitud del término "emprendimiento". 2.2. Emprendimiento, ¿crecimiento y

\footnotetext{
Universidad Pública de Navarra, Departamento de Trabajo Social laureano.martinez@unavarra.es

2 Universidad Pública de Navarra, Departamento de Trabajo Social patricia.amigot@unavarra.es
} 
creación de empleo? 3. El emprendimiento más allá del empleo: ahondando en el "trabajo sobre sí". 3.1. El supuesto del sujeto "deficitario". 3.2. La producción de una subjetividad emprendedora. 4. Conclusiones. 5. Referencias bibliográficas.

Cómo citar: Martínez Sordoni, L.; Amigot Leache, P. (2018) “Tensiones entre el emprendimiento y el trabajo autónomo en las políticas europeas de empleo: un nuevo capítulo en la relación entre el "trabajo como empleo" y el "trabajo sobre sí"”. Cuadernos de Relaciones Laborales, 36(2), 245-254.

\section{Introducción}

Tanto a nivel europeo como en el Estado español, las actuales medidas de promoción del emprendimiento incluyen el fomento del empleo autónomo entre sus principales instrumentos (Alameda Castillo, 2016; Fernández García, 2016; Luján Alcáraz y Ferrando García, 2016; Suárez Corujo, 2017). Esto se encuentra plasmado en el Plan de Acción sobre emprendimiento 2020 de la Comisión Europea (2013) y en la Ley 14/2013 de apoyo a los emprendedores y su internacionalización en España (BOE, 2013). Si bien las figuras del "emprendimiento" y el "trabajo autónomo" cuentan con una larga historia, la asociación entre una y otra no ha sido una constante a lo largo del tiempo. Por ello, este artículo propone un enfoque crítico sobre la forma en que dicha asociación queda plasmada en el diseño de las medidas de emprendimiento en el espacio comunitario europeo y su correspondencia en el Estado español.

Estas medidas son producto de un largo proceso de transformación económica y social que ha afectado a las nociones de "emprendimiento" y de "emprendedor" En la bibliografía económica del periodo industrial, con la teoría schumpeteriana como una de las principales referencias, el concepto de emprendedor remitía a un tipo particular de agente económico que asumía el riesgo de innovar en un contexto productivo dominado por las grandes organizaciones (Nightingale y Coad, 2013; Pérez Vila, 2007). A diferencia de lo que ocurre actualmente, Schumpeter señalaba el carácter "excepcional" de esta figura en el conjunto de la población: "actuar con confianza más allá del horizonte de lo conocido y vencer la resistencia del medio requiere aptitudes que solo están presentes en una pequeña fracción de la población y que definen tanto el tipo como la función del emprendedor" (Schumpeter, 1942: 132). En ese sentido, era una figura numéricamente reducida y con una función referida específicamente al espacio económico.

Por su parte, desde el punto de vista de las políticas productivas y de desarrollo económico, hasta entrados los años ochenta del siglo XX el emprendimiento estuvo incluido fundamentalmente en las políticas de desarrollo industrial, en un contexto en el que se relegaba a la marginalidad a los pequeños emprendimientos por considerarlos ineficientes comparados con las grandes empresas (Audretsch, 2007). Si bien el rol de los pequeños emprendimientos logró sostenerse en algunos sectores de la producción, lo cierto es que la tendencia descendente en su participación en

\footnotetext{
A lo largo del artículo se hará referencia al "emprendimiento" entendido como fenómeno económico y productivo. En las medidas analizadas se vincula a distintas formas de empresas o negocios. Por su parte, el concepto "emprendedor" remite al individuo y sus características personales o subjetivas (iniciativa, autonomía, asunción de riesgos, creatividad, etc.).
} 
el producto y en el empleo fue generalizada hasta mediados de la década de 1970 (Valdaliso Gago y López, 2007).

En la actualidad, el impulso del emprendimiento y la iniciativa emprendedora como política de crecimiento y empleo forma parte de las transformaciones del capitalismo de finales de los años setenta del siglo XX. Estas transformaciones han afectado tanto a la producción como al empleo. En lo que respecta a la producción, el advenimiento del capitalismo flexible, la extensión del fenómeno de la tercerización (outsoursing), el crecimiento del sector de los servicios y la necesidad de modelos productivos adaptables a contextos cambiantes han transformado las condiciones que habían relegado a los pequeños emprendimientos a un rol secundario por resultar ineficientes, permitiendo que sean señalados como los principales agentes dinamizadores de la economía (Audretsch, 2007). Del lado del empleo, se ha observado un desplazamiento del régimen de empleo salarial hacia lo que puede denominarse un "régimen de empleo empresarial" (Prieto Rodríguez, 2013). Este régimen ha sido producto de sucesivas reformas de corte neoliberal de los mercados de trabajo, sustentadas en los principios de la "flexibilidad" laboral y la libertad de mercado. Las nuevas formas de producción conjugadas con las reformas del mercado de trabajo han resultado en el debilitamiento de la centralidad del empleo típico y en la expansión de actividades realizadas en régimen de autonomía (Bureau y Corsani, 2014; Valdés Alonso, 2000).

Al mismo tiempo, a medida que el desempleo se volvía un fenómeno estructural, han ganado peso las políticas de empleo del lado de la oferta (supply side policies), caracterizadas por una individualización de la responsabilidad frente a los riesgos sociales. En el caso europeo, las medidas de emprendimiento se han incorporado al conjunto de estrategias de empleo basadas en los paradigmas de la empleabilidad, la activación y la flexiguridad (Fernández Rodríguez y Serrano Pascual, 2014). En ese sentido, el emprendimiento no pertenece ya solo al ámbito del desarrollo industrial, sino que se ha extendido hacia las políticas activas de empleo -formación y fomento del empleo- (Luján Alcáraz y Ferrando García, 2016) y las políticas educativas (Eurydice, 2015). Es por ello posible afirmar que, si bien la idea del emprendedor como agente económico que motoriza el crecimiento no es nueva, en los últimos años ha tomado un impulso y una fisonomía específica que permiten diferenciarla de momentos anteriores y posibilitan interrogarla en su singularidad.

Los objetivos perseguidos con mayor frecuencia por las medidas de emprendimiento son: (i) creación de empleo, (ii) mejoras en la empleabilidad (iii) crecimiento económico, (iv) mayor competitividad e innovación de la economía (BOE, 2013; Comisión Europea, 2013a; Eurostat, 2012; MESS, 2013). Más emprendimiento conllevaría mejoras económicas, mayor innovación, mayor competitividad y creación de empleo. Para lograr esos objetivos, estas políticas se basan en dos grandes estrategias. Por un lado, en la necesidad de crear un entorno que promueva y facilite la apertura de "nuevas empresas", independientemente de que se trate de empresas individuales, pymes o grandes empresas. En este caso se hace referencia al "emprendimiento" como fenómeno económico y productivo. Por otro lado, que es necesaria una "revolución cultural" que permita "liberar el potencial del emprendimiento" (Comisión Europea, 2013b: 4) y que, entre otras cuestiones, lleve a las personas a optar por el empleo autónomo frente a la opción por el empleo asalariado. Este supuesto afecta la dimensión subjetiva de los individuos (sus aspiraciones, sus emo- 
ciones, sus valores, sus preferencias, etc.), que suele ser referida como "espíritu", "mentalidad", "cultura" o "iniciativa" emprendedora.

El objetivo de este artículo es poner de manifiesto que las medidas adoptadas bajo estos supuestos conllevan dos movimientos estrechamente relacionados. Por un lado, como se desarrolla en el apartado 2, se destaca una tendencia individualizadora de las dinámicas laborales, que queda reflejada en la preeminencia del emprendimiento como empleo autónomo. Desde el punto de vista económico, el empleo autónomo es una unidad cuyo aporte al crecimiento y a la generación de nuevos empleos no siempre es significativa y, desde el punto de vista jurídico, supone una "huida del derecho del trabajo" que hace emerger procesos de precarización (Rodríguez-Piñero, Casas, y Valdés Dal-Ré, 2013). Esto genera una contradicción entre los objetivos de las políticas antes señalados y los instrumentos que ponen en acción para alcanzarlos. Por otro lado, en el apartado 3, se muestra que la estrategia de desplazamiento de la "cultura del empleo asalariado" por una "cultura emprendedora" se caracteriza por fomentar un modelo de sujeto que lleva la acción de "emprender" más allá del dominio del trabajo como empleo hacia el dominio del "trabajo sobre sí", lo que conduce a un modelo de sujeto "empresario de sí mismo" (Foucault, 2007; Vrancken y Macquet, 2012). Se trata, por tanto, de interrogar al emprendimiento en el cruce entre la regulación del trabajo y la construcción de sujetos trabajadores (Amigot Leache y Martinez, 2013; Crespo, Prieto, y Serrano, 2009, Martínez Sordoni, 2017).

Partiendo del concepto de europeización discursiva (Guillén y Álvarez, 2004; Pasquier, 2005), que remite a la coordinación entre distintos niveles gubernamentales de elementos "cognitivos y normativos" (paradigmas de políticas públicas, conceptos, teorías, mediciones estadísticas, "buenas prácticas”), el artículo se basa en el análisis de un conjunto de documentos (planes, programas, normativas, informes técnicos, discursos expertos) correspondientes al periodo de la Estrategia de Lisboa (2000-2010) y de la Estrategia Europa 2020 (2010 a la actualidad), que se encuentran coordinados entre las instituciones europeas y el Estado español y de los que se recogen algunos de los núcleos discursivos más relevantes ${ }^{4}$. Para abordar estos documentos, se conjuga una dimensión analítica, en la que se presentan una serie extractos seleccionados en función de su contenido informativo sobre el tema analizado, y una dimensión comprensivo interpretativa en la que intentamos comprender, a partir de los segmentos seleccionados, cómo el discurso europeo sobre el emprendimiento opera como un dispositivo de gobierno organizador de una sociedad y una subjetividad emprendedora.

El artículo resume algunos de los puntos abordados en una investigación de tesis doctoral (Martínez Sordoni, 2017), con un corpus de más de 100 documentos correspondientes a distintos niveles gubernamentales (Europeo y estatal), así como a "discursos expertos” provenientes de las ciencias económicas e instituciones público-privadas que promueven la iniciativa emprendedora. 


\section{Las tensiones entre el autoempleo y la creación de empresas}

\subsection{Problemas en torno a la amplitud del término "emprendimiento"}

A diferencia del carácter "excepcional" y cuantitativamente reducido que definía al emprendedor del periodo industrial, actualmente es posible señalar una ampliación de los sujetos interpelados por el discurso del emprendimiento. Las políticas de activación para el empleo se proponen "llegar a las mujeres, los mayores, los inmigrantes, los desempleados y los jóvenes" (Comisión Europea, 2013b: 24). Es decir, se dirigen a los grupos "con especiales dificultades de integración en el mercado de trabajo" (BOE, 2015). Al mismo tiempo, la llamada a devenir emprendedores no se limita a quienes se encuentran en situación de desempleo o inactividad, sino que se incorpora en todas las instancias educativas (Comisión Europea, 2013b; Eurydice, 2015) y se dirige a personas que trabajan en relación de dependencia, bajo la figura del "intraemprendedor" (quien da origen a un emprendimiento en el seno una empresa) (Comisión Europea, 2003). En suma, las medidas sostienen que los nuevos riesgos sociales, "asociados a la transición hacia una economía postindustrial del conocimiento y de servicios" (Comisión Europea, 2007b: 6), hacen que el espíritu emprendedor no sea propio de un perfil definido de individuos sino "un factor clave para todos" (Comisión Europea, 2006: 4). Este criterio se ve reflejado en la definición que en su Artículo 3 ofrece la Ley 14/2013, de apoyo a los emprendedores y su internacionalización: "se consideran emprendedores aquellas personas, independientemente de su condición de persona física o jurídica, que desarrollen una actividad económica empresarial o profesional, en los términos establecidos en esta Ley" (BOE, 2013).

A esta diversidad de sujetos que son señalados como potenciales emprendedores, se suma la gran amplitud y diversidad de "emprendimientos" que pueden resultar de sus iniciativas. Si nos atenemos a los términos asociados al emprendimiento en los documentos analizados, encontramos que se vincula con el alta de "nuevas empresas" o la puesta en marcha de nuevos negocios, independientemente de su forma jurídica y tamaño. Tal como lo define el GEM (Global Entrepreneurship Monitor): "cualquier intento de puesta en marcha o creación de un nuevo negocio, desde el autoempleo a la expansión o desarrollo de un negocio o empresa ya existente, por un individuo, equipo de personas o compañía ya establecida" (de Castro, Justo, y Maydeu Olivares, 2008: 31). Se trata de una definición amplia que incluye cualquier iniciativa sin distinción en términos de volumen de negocio, de años de actividad, personas empleadas o capital inicial invertido.

Como ejemplo de estos criterios traducidos a las políticas públicas, el portal del Ministerio de Economía, Industria y Competitividad de España ofrece información para que quien quiera emprender pueda elegir la forma jurídica de una futura empresa. Se basa en tres grandes categorías: responsabilidad (limitada o ilimitada), número de socios (uno, dos, tres o más) y el capital social (sin mínimo legal, entre $3.000 €$ $-59.999 €$, entre $60.000 €-120.000 €$ o más de $120.000 €$ ). De esta manera, se contabilizan veinte formas jurídicas con características muy distintas; desde el trabajador autónomo o "empresario individual", forma jurídica compuesta por un solo socio cuya responsabilidad es ilimitada y que no requiere un capital mínimo para comen- 
zar, hasta la Sociedad de Garantía Recíproca, para la cual se necesitan un mínimo de 150 socios, que tienen una responsabilidad limitada y que requiere un capital mínimo de 10 millones de euros 5 .

Estas definiciones y principios tienen sus bases en la teoría económica. En términos del economista David Audretsch, uno de los "expertos" de referencia en las medidas europeas, muchos de los elementos que constituyen la iniciativa emprendedora o "capital emprendedor" de una sociedad desafían la cuantificación, por tratarse de un fenómeno multifacético y heterogéneo. Sin embargo, señala el autor, el capital emprendedor tiene una singularidad, que es "la puesta en marcha de nuevos negocios". Por ello se propone el uso de "la tasas de puesta en marcha de nuevas empresas como indicador proxy de la variable inobservable o latente" (Audretsch, 2009: 37).

Es decir, las medidas no se dirigen a un tipo de emprendimiento en particular, sino que se caracterizan por incluir una amplia diversidad de agentes económicos. Lo que nos interesa destacar es que, en función de distintas estrategias nominativas, el término "emprendimiento" aparece como un "significante vacío" o "significante flotante" (Kenny y Scriver, 2012) que conjuga diversos significados: PYME -con la diversidad de tipos de empresa que cada categoría encierra-, trabajadores autónomos, empresas unipersonales, sociedades laborales, etc. La diversidad de potenciales sujetos emprendedores y la pluralidad de resultados posibles en términos de emprendimientos resultan problemáticas desde el punto de vista de las dinámicas económicas y laborales que generan.

Partiendo de distintas situaciones posibles -desempleo, inactividad, empleo asalariado, etc.- no es lo mismo una transición al emprendimiento como trabajador autónomo que como socio en una empresa innovadora de base tecnológica (EIBT) con personas empleadas a cargo. A este respecto, puede establecerse analíticamente una distinción en función de lo que se señala como finalidad principal de "emprender": el emprendimiento como creación de una empresa innovadora con potencial de crecimiento y el emprendimiento como autoempleo. Es decir, es posible diferenciar el emprendimiento según sea su principal motivación la de crear empleo para sus miembros o la de invertir un capital que genere ganancias y crecimiento económico (Nightingale y Coad, 2013). El autoempleo puede ser entendido como un acceso al empleo a partir de la creación por parte de los individuos de su propio puesto de trabajo. Las iniciativas dirigidas a fomentar esta práctica son numerosas y se vienen implementando por diversos organismos gubernamentales desde hace décadas. Dichas iniciativas pueden adoptar variadas formas: apoyo al trabajo autónomo, incentivos a la economía social o estímulos a la constitución de pequeñas empresas (Comisión Europea, 2013b; MESS, 2013). En todos los casos se pretende que uno o varios individuos -generalmente en situación de desempleo- dejen esa condición a partir de la creación de su propio puesto de trabajo.

El autoempleo individual se refiere a la puesta en marcha de una actividad productiva o de servicios por parte de una persona que la realizará generalmente de forma particular, como empleo autónomo, o bien contratando a otras personas, con lo que se convierte jurídicamente en empleador. El autoempleo colectivo o empleo asociado consiste en la asociación del trabajo de varios individuos mediante la puesta en marcha de una actividad de producción de bienes o servicios, generalmente a

Véase: http://www.ipyme.org/es-ES/DecisionEmprender/FormasJuridicas/Paginas/FormasJuridicas.aspx [consulta el 12 de diciembre de 2017]. 
través de la constitución de una sociedad, que será la titular de la empresa y dará empleo a los socios (García Jiménez, 2008). Los emprendimientos puestos en marcha y gestionados por trabajadores a través de la autoorganización de la actividad productiva, suelen tener como objetivo principal y estratégico el mantenimiento de los puestos de trabajo. En el caso de no tener principalmente un interés especulativo o de ganancia, sino el de garantizar el empleo a sus miembros, suelen situarse dentro del concepto de economía social.

Pero no toda iniciativa emprendedora, aunque sea de pequeña dimensión, puede ser calificada de autoempleo. Una iniciativa emprendedora puede corresponderse con una actividad inversora - con capital propio o en préstamo, de forma individual o colectiva- donde la generación de empleo es algo instrumental o "secundario", siendo el objetivo principal el desarrollo de un proyecto de negocios para la obtención de ganancias del capital invertido. Las sociedades que dan forma jurídica al tipo de asociaciones de autoempleo reúnen una serie de características que las diferencian de las empresas que invierten capital. Tienen, además, una dinámica muy distinta en cuanto a su desempeño económico, a la generación de empleo y aporte a la innovación (Nightingale y Coad, 2013).

Sin embargo, desde el punto de vista del diseño de las medidas analizadas, la distinción entre el objetivo de creación y preservación de puestos de trabajo y el de retorno de una inversión de capital como finalidades diferenciadas queda difuminada bajo la consigna "crecimiento económico y creación de empleo", como si se tratara de un contínuum evidente e indistinto. A causa de ello, se produce una indiferenciación entre las medidas que señalan al emprendimiento como núcleo de un modelo de desarrollo y crecimiento innovador y los instrumentos de fomento del autoempleo propios de las medidas de activación (Román, Congregado, y Millán, 2013).

\subsection{Emprendimiento, ¿crecimiento y creación de empleo?}

La amplitud de formas que caben bajo el término "emprendimiento" hace que los instrumentos propuestos en las medidas resulten problemáticos de cara a sus objetivos (creación de empleo, crecimiento económico, mejora de la competitividad, etc.). Según datos del Directorio Central de Empresas (DIRCE) del Instituto Nacional de Estadísticas de España (INE), de las 3.282.346 empresas existentes en España al 1 de enero de 2017 (incluyendo personas físicas, mayoritariamente autónomos) en torno al 55\% (1.823.250) no tienen personas empleadas y un 27\% (894.179) tienen entre 1 y 2. Estos porcentajes son similares a los años previos a la crisis. A esto debe sumarse que de las 419.504 empresas dadas de alta durante todo 2016, un 76\% (318.886) fueron dadas de alta sin personas empleadas y casi un $22 \%$ (91.389) surgió con entre 1 y 5 empleados.

Datos similares se encuentran si se toma como referencia la información sobre la dinámica emprendedora ofrecida por el proyecto GEM en su informe para España de 2016 (Tabla 1). Allí puede verse que los nuevos emprendedores sin empleados representan el 52,4\% para los negocios en fase inicial y el 38\% para los negocios consolidados para el año 2016, mientras que los nuevos emprendedores con entre 1 y 5 empleados representan el 38,9\% para los negocios en fase inicial y $49,3 \%$ en el caso de los emprendimientos consolidados. Si bien pueden notarse variaciones desde el inicio de la crisis en 2007, lo cierto es que existe una clara tendencia de empren- 
dimientos con menos de cinco o con ninguna persona asalariada, que en conjunto se encuentran siempre por encima del $80 \%$ en el caso de los negocios consolidados y en torno al $90 \%$ en los de fase inicial.

Tabla 1: Distribución de los emprendedores en fase inicial y emprendedores consolidados en España, por el tamaño en empleo de sus proyectos de negocio entre 2007 y 2016.

\begin{tabular}{|c|c|c|c|c|c|c|c|c|}
\hline & \multicolumn{4}{|c|}{$\begin{array}{l}\text { Negocios en fase inicial } \\
\text { (de } 0 \text { a } 42 \text { meses })\end{array}$} & \multicolumn{4}{|c|}{$\begin{array}{l}\text { Negocios consolidados } \\
\text { (más de } 42 \text { meses) }\end{array}$} \\
\hline Año & \multicolumn{4}{|c|}{$\mathrm{N}^{\mathrm{o}}$ de personas empleadas } & \multicolumn{4}{|c|}{$\mathrm{N}^{\mathrm{o}}$ de personas empleadas } \\
\hline & 0 & $1-5$ & $6-19$ & $\begin{array}{l}20 \text { y } \\
\text { más }\end{array}$ & 0 & $1-5$ & $6-19$ & $\begin{array}{l}20 \text { y } \\
\text { más }\end{array}$ \\
\hline 2007 & $43,5 \%$ & $42,5 \%$ & $11,6 \%$ & $2,5 \%$ & $49,8 \%$ & $34,3 \%$ & $11,9 \%$ & $4,0 \%$ \\
\hline 2008 & $40,0 \%$ & $43,1 \%$ & $14,0 \%$ & $2,9 \%$ & $49,5 \%$ & $33,8 \%$ & $12,7 \%$ & $4,0 \%$ \\
\hline 2009 & $40,7 \%$ & $49,8 \%$ & $7,3 \%$ & $2,2 \%$ & $35,5 \%$ & $49,4 \%$ & $11,3 \%$ & $3,8 \%$ \\
\hline 2010 & $66,0 \%$ & $27,9 \%$ & $4,9 \%$ & $1,2 \%$ & $39,7 \%$ & $48,6 \%$ & $8,7 \%$ & $2,9 \%$ \\
\hline 2011 & $70,7 \%$ & $25,0 \%$ & $3,3 \%$ & $1,0 \%$ & $39,2 \%$ & $46,8 \%$ & $10,1 \%$ & $4,0 \%$ \\
\hline 2012 & $57,1 \%$ & $36,4 \%$ & $5,7 \%$ & $0,8 \%$ & $47,4 \%$ & $39,7 \%$ & $9,9 \%$ & $2,9 \%$ \\
\hline 2013 & $52,2 \%$ & $39,6 \%$ & $5,1 \%$ & $3,2 \%$ & $49,7 \%$ & $38,1 \%$ & $9,1 \%$ & $3,1 \%$ \\
\hline 2014 & $54,7 \%$ & $37,1 \%$ & $6,5 \%$ & $1,7 \%$ & $40,3 \%$ & $45,4 \%$ & $10,9 \%$ & $3,4 \%$ \\
\hline 2015 & $50,5 \%$ & $44,9 \%$ & $4,1 \%$ & $0,5 \%$ & $42,8 \%$ & $46,7 \%$ & $7,5 \%$ & $2,9 \%$ \\
\hline 2016 & $52,4 \%$ & $38,0 \%$ & $6,4 \%$ & $3,2 \%$ & $38,9 \%$ & $49,3 \%$ & $8,7 \%$ & $3,1 \%$ \\
\hline
\end{tabular}

Fuente: elaboración propia a partir de GEM (2016).

Si nos centramos en señalar la convención que incluye bajo una misma categoría de "emprendimiento" las altas de diverso tipo de empresas, los datos pueden leerse de la siguiente forma: puede notarse que los emprendimientos en España son, en su mayoría, personas físicas (autónomos) sin empleados a cargo o con menos de 5 empleados.

En este sentido, numerosos analistas han señalado que, a causa de la amplitud del término "emprendimiento", las conclusiones sobre el impacto positivo de este en la economía y el empleo son ambivalentes (Congregado, Golpe, y Carmona, 2010; Nightingale y Coad, 2013; Shane, 2009). El empleo autónomo conforma un grupo sumamente heterogéneo y sólo una minoría contrata personas a cargo o crea negocios que generan innovación y riqueza. Por ello, más autoempleo y más trabajadores autónomos no es un indicador necesariamente positivo (Congregado, Golpe, y Carmona, 2010). Como ha indicado Blanchflower (2004), "más" puede no ser "mejor". 
Por su parte, un análisis longitudinal en España sobre las medidas de emprendimiento y autoempleo ha puesto de manifiesto la ambivalencia de estas políticas si se tiene en cuenta el punto de origen de la transición al empleo autónomo (Millán, Congregado, y Román, 2012). Cuando el origen es el desempleo, los emprendimientos tienen una alta probabilidad de fracaso. Los autores sugieren un impacto positivo de la educación formal y la experiencia previa en el mercado laboral sobre la supervivencia de los emprendimientos, lo que habla de perfiles específicos como potenciales personas emprendedoras creadoras de empleo y riqueza económica, y no de la totalidad de la población, y mucho menos de los grupos "con especiales dificultades de integración en el mercado de trabajo", tal como lo proponen las actuales medidas.

Desde luego que la idea de centrarse en la "cantidad" de nuevas empresas como indicador de una dinámica emprendedora no está exenta de discusiones. De hecho, a medida que en los últimos años la calidad de los datos ha mejorado y los métodos se han hecho más sólidos, las investigaciones desde las disciplinas económicas han pasado de una valoración positiva sobre el número de emprendimientos hacia conclusiones más sobrias (Nightingale y Coad, 2013). No obstante, la formulación de políticas en la mayoría de los países sigue asumiendo casi universalmente que una mayor tasa de altas de empresas es un dato positivo. Al respecto, Scott Shane, reconocido economista en materia de emprendimiento, es tajante a la hora de valorar la amplitud de tipos de empresas contempladas en las medidas públicas:

Quienes formulan las políticas a menudo piensan que la creación de empresas (start-up) va a transformar regiones económicamente deprimidas, generar innovación y crear puestos de trabajo. Esta creencia es errónea porque la típica start-up puesta en marcha no es innovadora, crea pocos puestos de trabajo y genera poca riqueza. Conseguir el crecimiento económico y la creación de puestos de trabajo a partir del emprendimiento no es un juego de números. Se trata de fomentar la formación de alta calidad y las empresas de alto crecimiento. Quienes diseñan las políticas deberían dejar de subvencionar la formación de la típica start-up y enfocarse en el subconjunto de empresas con potencial de crecimiento (Shane, 2009: 141. La traducción es nuestra).

Pero no solo el diseño de las medidas entra en contradicción con sus objetivos de crecimiento económico y creación de empleo, sino que puede conducir a la creación de empleos precarios (Ballester Pastor, 2016; Briales Canseco, 2017). La formulación de las políticas de emprendimiento parecería conducir a lo que Supiot (1997) señalaba como una de las formas de ruptura del estatuto salarial: la privación al trabajador del estatuto de asalariado, cuyo ejemplo paradigmático es la incitación -ya sea a los desempleados o a los asalariados- a devenir trabajadores autónomos. Esto genera una "huida del derecho del trabajo", tal como sostienen un conjunto de investigaciones que han indagado la dimensión jurídica de las políticas de emprendimiento adoptadas en el Estado español (Casas Baamonde, 2013; Molina Navarrete, 2013; Rodríguez-Piñero, Casas y Valdés Dal-Ré, 2013).

Desde el punto de vista del derecho, estos trabajos señalan que las figuras de apoyo a la actividad emprendedora en general, y particularmente las propuestas por la Ley de emprendedores en España (BOE, 2013), son de carácter marcadamente mercantil. En efecto, si se contemplan los instrumentos jurídicos ofrecidos por la 
regulación, nos encontramos con una huida del ámbito del derecho del trabajo hacia el empleo autónomo investido jurídicamente como "actividad emprendedora / sujeto emprendedor". Este instrumento comporta un desplazamiento desde los contratos de trabajo hacia los contratos de servicios civiles o mercantiles -y a su vez de la nómina salarial a la factura por servicios-, lo cual conduce a que las cotizaciones de los empleadores se desplacen hacia las "cuotas de autónomos". Como es sabido, este tipo de relaciones laborales implican que es la persona que trabaja quien debe asumir la mayor parte de los riesgos del mercado de trabajo y la economía y supone una clara remercantilización del trabajo (Alonso, 2011; Ballester Pastor, 2016; Molina Navarrete, 2013). Esto señala una dinámica de las relaciones laborales en la cual la ruptura de los mecanismos colectivos que garantizan la condición salarial está dejando lugar a un vínculo que supone la igualdad de los contratantes al estilo del "contrato de servicios" del liberalismo decimonónico.

Pero como hemos dicho al principio, es preciso indagar el discurso europeo del emprendimiento más allá de sus objetivos explícitos de creación de empleo y generación de riqueza. Por ello, luego de haber señalado las tensiones entre emprendimiento y trabajo autónomo desde el punto de vista del empleo, es preciso abordar el modelo de sujeto-trabajador que se corresponde con la narrativa que señala un déficit de emprendimiento a nivel de la población e intenta universalizar el "emprender" como el modo de acción más valorado socialmente.

\section{El emprendimiento más allá del empleo: ahondando en el "trabajo sobre sí"}

Desde el punto de vista de las características subjetivas, la amalgama entre emprendimiento y trabajo autónomo encuentra su fundamento en uno de los principales postulados de la teoría económica. Se trata del principio que señala que uno de los rasgos psicológicos de las personas emprendedoras "es la creencia innata en que su rendimiento depende, en gran medida, de sus propias acciones en lugar de factores externos. En el dominio de la psicología se llama a esto 'tener un alto locus de control interno" (Parker, 2004: 76). Si bien el uso que el discurso económico realiza de la psicología no está exento de críticas, lo cierto es que ha logrado construir una imagen "heroica" de la personalidad emprendedora que se caracterizaría por una percepción de que los acontecimientos de su vida dependen de sus propias acciones más que de factores externos, como la estructura económica y la regulación del mercado de trabajo. Y bajo el supuesto de que el empleo autónomo ofrecería mayor "libertad" y "discrecionalidad" a los individuos que el empleo por cuenta ajena, se concluye en que las personas emprendedoras, caracterizadas por un locus de control interno, se inclinarían por el empleo autónomo antes que por el empleo en relación de dependencia (Grilo y Thurik, 2004; Parker, 2004).

Las actuales políticas de promoción del emprendimiento -y he aquí uno de los núcleos argumentativos para entender las actuales medidas en la materia-invierten los términos de este postulado y proponen incentivar la opción por el empleo autónomo como forma de promover el espíritu emprendedor y la apertura de nuevas empresas. Como si la "liberación" del potencial emprendedor pasara por una suerte de "abandono" del referencial del empleo asalariado y de todo su entramado de derechos y protecciones colectivas -las cuales pasan a ser caracterizadas como "obstácu- 
los" o "barreras"- en beneficio del trabajo autónomo, al cual se asocian valoraciones vinculadas a la "internalidad" como la autonomía, la independencia, la libertad y la creatividad (Comisión Europea, 2007a; MESS, 2013).

\subsection{El supuesto del sujeto "deficitario"}

Desde el punto de vista argumentativo, las medidas analizadas sostienen que el desempleo y la falta de crecimiento económico se deben en buena parte a un déficit de iniciativa emprendedora en la población. En este sentido, las medidas de emprendimiento se inscriben en una concepción del desempleo centrada en supuestos déficits individuales más que en las dinámicas estructurales (Crespo Suárez y Serrano Pascual, 2013). Por poner un ejemplo, la Ley 14/2013, de 27 de septiembre, de apoyo a los emprendedores señala:

...uno de los graves problemas de la economía y sociedad española es la alta tasa de desempleo juvenil [...] Las causas de ello hay que buscarlas, además de en algunas deficiencias que han venido caracterizando a nuestro modelo de relaciones laborales, en la ausencia de una mayor iniciativa emprendedora entre los más jóvenes que haya llevado, ante la falta de oportunidades de trabajo por cuenta ajena, a unos mayores niveles de autoempleo capaces, a su vez, de generar más empleo (BOE, 2013: 78791).

Ahora bien, asumiendo que la construcción del argumento que justifica las políticas es reflejo de una disputa por dotar de sentido al ordenamiento social, es preciso interrogarse sobre los instrumentos que permiten construir la idea de un "déficit" en materia de iniciativa emprendedora como problema público, así como sobre las prácticas propuestas para revertir la situación definida como problemática. Aquí entran en juego un conjunto de instrumentos de medición estadística, que se encuentran armonizados a nivel europeo, producto del proceso de europeización de las políticas públicas (Guillén y Álvarez, 2004; Pasquier, 2005). Entre los principales instrumentos pueden señalarse los siguientes: (i) Eurostat/OCDE Entrepreneurship Indicators Programme; (ii) Flash Eurobarómetro n 354 : "Entrepreneurship" y (iii) y los informes del Global Entrepreneurship Monitor (GEM) ${ }^{6}$.

En correspondencia con los postulados de la teoría económica señalados más arriba, la medición del nivel de cultura emprendedora de una sociedad se construye, entre otras variables, en torno a la preferencia de la población por el empleo autónomo (CIS, 2012; Comisión Europea, 2013b; Eurostat, 2012; Grilo y Thurik, 2004). Como han puesto de manifiesto los trabajos de la sociología de la cuantificación (Desrosières, 2014), la medición de los fenómenos sociales no es una práctica "neutral", sino que tiene efectos prácticos sobre la realidad, en tanto segmenta y objetiva procesos y experiencias sociales que pretenden transformarse. El argumento estadístico

El Flash Eurobarómetro n 354: "Entrepreneurship" ha servido de modelo en España para la encuesta del Centro de Investigaciones Sociológicas (CIS, 2012) sobre Actitudes de la juventud en España hacia el emprendimiento. En el caso de los informes del Global Entrepreneurship Monitor, si bien son llevados a cabo por organismos independientes de las instituciones europeas, son una referencia permanente en las medidas comunitarias sobre emprendimiento en los últimos años (Comisión Europea, 2013b). 
confiere objetividad y legitimidad a la asociación. Pone a disposición de los actores sociales argumentos que son resistentes a las críticas, por su legitimidad como saber experto, y creadores de un sentido común, en cuanto incita a utilizar habitualmente determinado tipo de información por sobre otros (Desrosières, 2014).

Desrosières propone distinguir entre convención y medición dentro del proceso de cuantificación. La cuantificación es entendida como "expresar y hacer existir sobre una forma numérica aquello que anteriormente se expresaba en palabras y no en números" (Desrosières, 2014: 38). Cuantificar supone que sean elaboradas y explicitadas de antemano un conjunto de "convenciones", que implican prácticas discursivas (conceptos, teorías, argumentos, etc.), para llegar a una composición numérica. La medición, por su parte, supone la comparación de la realidad social objetivada con la convención previamente establecida. Es precisamente la convención que asocia la iniciativa emprendedora al empleo autónomo la que debe ser problematizada, ya que las "mediciones" del espíritu emprendedor o la iniciativa emprendedora en la población no son simples instrumentos que reflejan o constatan una situación dada, sino que en sus "convenciones" delimitan un conjunto de factores sobre los que se deberá intervenir con el objetivo de aumentar los índices de iniciativa emprendedora. Es decir, son instrumentos de poder en una estrategia de regulación de los sujetos sociales. Para poner un ejemplo entre muchos posibles, detengámonos en la siguiente afirmación del Plan sobre Emprendimiento 2020, de la Comisión Europea (2013). En el apartado titulado "Nuestro reto: más emprendedores para Europa", se presenta el siguiente argumento, basado en datos del Flash Eurobarómetro 354: Emprendimiento.

Las nuevas empresas, y especialmente las PYME, representan la fuente más importante de nuevos empleos: crean más de cuatro millones de puestos de trabajo al año en Europa. Pero el motor de esa recuperación está fallando: desde 2004, la proporción de personas que prefieren trabajar por cuenta propia antes que convertirse en asalariadas ha disminuido en veintitrés de los veintisiete Estados miembros de la UE. Mientras que el trabajo por cuenta propia era hace tres años la primera opción del $45 \%$ de los europeos, este porcentaje ha bajado ahora hasta el $37 \%$. En cambio, en los Estados Unidos y China esta proporción es mucho mayor: el $51 \%$ y el $56 \%$ respectivamente (Comisión Europea, 2013b: 4).

La anterior cita toma su fundamento de los resultados del Eurobarómetro, en respuesta a la pregunta: si pudiera elegir entre diferentes tipos de empleo, ¿cuál preferiría? Cuyas opciones de respuesta son: a) un empleo por cuenta ajena; b) trabajar por cuenta propia (European Commission, 2012). La convención que asocia "trabajo por cuenta propia" con la "creación de nuevas empresas" permite construir el argumento que señala un "fallo" en el "motor" de la recuperación económica.

Como señala Robert Salais (2010), es frecuente encontrar en los argumentos estadísticos de las políticas sociales y de empleo que el enunciador hace como si fuera posible pasar, sin interrogar la legitimidad del pasaje, de la proposición "A" a la proposición "B”. En el ejemplo citado, esto sería: A) “El motor de esa recuperación está fallando" y B) "la proporción de personas que prefieren trabajar por cuenta propia antes que convertirse en asalariadas ha disminuido". El primer enunciado nos remite al universo del problema del desempleo y el crecimiento en Europa. En el segundo, se hace referencia a una cultura que prefiere el empleo asalariado por encima del em- 
prendimiento / empleo autónomo (hay "fallos" porque las personas prefieren ser asalariadas), lo cual implica asumir acríticamente el vínculo "trabajo por cuenta propia / emprendimiento". Por tanto, entre los enunciados "A" y "B" no existe una relación de causalidad necesaria, sino que "la fuerza y la eficacia política de la enunciación reposa en la ausencia de cuestionamiento público (y colectivo) sobre la legitimidad que hay en identificar las proposiciones A y B y sobre el sentido que cada una tiene" (Salais, 2010: 130).

La relevancia de cuestionar este criterio radica en que a partir del mismo se justifican el conjunto de medidas educativas y de formación emprendedora, entre las cuales se encuentra el fomento del empleo autónomo. Es decir, aquello que el argumento estadístico, apoyado en el discurso económico, señala como un "determinante del emprendimiento", las políticas públicas lo transforman en objeto de intervención. De esta forma, las preferencias de los individuos con respecto al tipo de empleo devienen blanco de las estrategias de poder. La opción por el empleo por cuenta ajena resulta deslegitimada y se realza la opción por el empleo autónomo. En concreto, uno de los principales objetivos de una educación emprendedora es: “...fomentar la sensibilización de los estudiantes sobre el empleo autónomo y el espíritu empresarial de como opciones viables para su futura carrera" (European Commission, 2006: 15) o "fomentar el empleo autónomo y la creación de empresas mediante la educación, la formación y la información..." (MESS, 2013: 57). La identificación de los problemas del campo económico con la preferencia por el tipo de empleo más que un enunciado "descriptivo" debe ser entendido como un enunciado "performativo", es decir, un tipo de enunciado que pretende influir en las prácticas sobre las que enuncia, hacerlas concebibles y crear la representación y voluntad colectivas que pueden contribuir a hacerlas realidad (Bourdieu, 1985). En ese sentido, la "opción" por el empleo autónomo dependería menos de la "internalidad" de los sujetos que de la interiorización de las interpelaciones de los discursos hegemónicos, que pretenden moldear la subjetividad según los requisitos de flexibilidad, adaptabilidad, asunción de riesgos, autonomía e iniciativa, entre otros.

\subsection{La producción de una subjetividad emprendedora}

La necesidad de fomentar una subjetividad "emprendedora", "libre", "autónoma" y "creativa" es incitada por una potente retórica presente en múltiples espacios institucionales, que van desde las instancias públicas -educación y formación; servicios sociales y de empleo- hasta los medios de comunicación (European Commission, 2007). Esta retórica resalta la iniciativa, la cultura o la mentalidad emprendedora como características valoradas en todas las esferas sociales. Es decir, el discurso emprendedor contemporáneo no se caracteriza solamente por la amplitud de sujetos considerados potencialmente emprendedores sino que lleva la iniciativa emprendedora más allá del ámbito de la economía y el empleo: "el provecho de una educación en el espíritu emprendedor no se limita a que haya mayor cantidad de nuevas empresas, más innovadoras, y a que se creen más empleos" (Comisión Europea, 2006: 4). En la competencia de "sentido de la iniciativa y espíritu de empresa", afirma la Comisión, "se apoyan todas las personas, no sólo en la vida cotidiana, en casa y en la sociedad, sino también en el lugar de trabajo" (Comisión Europea, 2007: 11). 
Esta ampliación del alcance de la cultura emprendedora se corresponde con un proceso de "empresarialización" de la sociedad, es decir, de una extensión de las lógicas empresariales más allá de las fronteras del mundo de la economía y la producción, para pasar a ser un modelo válido en todos los ámbitos sociales (Laval y Dardot, 2013; Santos Ortega, 2014). En ese sentido, el hecho de proponer que el modelo del emprendedor "es un factor clave para todos" (Comisión Europea, 2009: 11) [...] "en la vida cotidiana, en casa y en la sociedad" (Comisión Europea, 2007: 3), nos lleva a sostener que no se trata simplemente de generar condiciones para la apertura de nuevos negocios que, de sostenerse en el tiempo, tenderían a generar nuevos puestos de trabajo, sino de poner en juego una serie de mecanismos cuyos efectos subjetivos sean acordes a los requerimientos de lo que el discurso económico llama una "sociedad emprendedora" (Audretsch, 2007).

Para "solucionar" las disfuncionalidades o los "déficits" a nivel de los conocimientos, las emociones o las actitudes que no concuerdan con las normas de una sociedad emprendedora, se pone en juego la construcción de un ideal a partir del cual movilizar identificaciones subjetivas hacia la valoración positiva de un tipo de conducta "emprendedora / autónoma", que opera de forma normativa y normalizadora. El emprendimiento responde, en este sentido, a un modelo de acción autónoma. Es decir, "el emprendedor es quien sabe conducirse por sí mismo" (Ehrenberg, 2010: 257). Desde el punto de vista de los mecanismos sociales, esto supone el paso de la autonomía como aspiración, en el marco de una ciudadanía social que otorgaba a los sujetos una protección frente a la asimetría de poder del mercado, hacia la autonomía como condición, propia de una ciudadanía económica basada en la participación en el mercado (Alonso, 2007). La autonomía no hace referencia a una mayor libertad de participación en las decisiones políticas, sino a un cambio en el espíritu de la acción que hace de la autonomía de los individuos, de la capacidad de transformarse a sí mismos y de actuar por sí mismos el valor supremo (Ehrenberg, 2010). En esta operación, se oculta que ese "conducirse a sí mismo" idealizado responde, más bien, a lógicas de acción de naturaleza heterónoma.

En un contexto de creciente incertidumbre, la necesidad de referentes profundiza la vulnerabilidad subjetiva y expone a los individuos a ideales que se corresponden con las demandas de una sociedad moldeada bajo los principios del mercado. La fuerte carga subjetiva de tipo emocional y volitiva que caracteriza al ideal del emprendedor opera como una instancia compensatoria ante el retroceso de las protecciones sociales y la emergencia de un entorno cambiante. En este sentido, el objetivo de las medidas de emprendimiento no apunta a la integración de las personas a través de derechos sociales universales, sino a través de una "intervención sobre sí mismos" (Vrancken y Macquet, 2012) con la finalidad de formar individuos de acuerdo con los valores de autonomía, responsabilidad, activación e iniciativa.

A partir del supuesto de un "potencial interno" que tendrían todas las personas y que sería accesible a través de un ejercicio reflexivo, el ideal del sujeto emprendedor señala el "trabajo sobre sí" que el sujeto debe realizar en su devenir emprendedor. De modo que, en base a la noción de performatividad del discurso, la práctica "devenir emprendedores" puede entenderse como la repetición de los actos indicados por el discurso hegemónico, el cual da por supuesta una "esencia" o "internalidad" que estaría "en todas las personas" y podría "liberarse", a partir de un trabajo del individuo sobre sí mismo. Es decir, la identificación de los individuos con el discurso emprendedor, su "aceptación" y la puesta en acción del "abandono" del referencial 
del empleo asalariado, implica para el sujeto realizar un trabajo sobre sí. Esto no es un acto único, sino una repetición performativa. No se deviene emprendedor "de una vez y para siempre", sino como producto de un trabajo reiterado que puede intensificarse o, contrariamente, interrumpirse.

Ese trabajo implica desarrollar y poner en juego las "competencias emprendedoras", no solo aquellas vinculadas a los conocimientos en gestión empresarial, sino fundamentalmente las competencias vinculadas a estados afectivos y emocionales como la autoconfianza, la ilusión y la pasión. Los textos de educación y formación sobre emprendimiento (European Commission, 2006; Eurydice, 2015) remiten a un trabajo del sujeto sobre sí mismo a partir de procesos mentales de cognición, afectivos o de autopercepción, como por ejemplo reconocer las habilidades personales, autoevaluar las propias competencias, automotivarse, tener autoconfianza, controlar el estrés y las emociones, tener deseo de asumir riesgos, entre otras. Los estados afectivos buscados se logran a partir de claves narrativas que capturan la experiencia con el fin de moldearla de forma voluntaria en base a la introspección y la reflexión sobre sí mismo (Zangaro, 2011). El objeto de la intervención serán así las preferencias, competencias, motivaciones, los deseos y las actitudes individuales, siendo el sujeto individual el blanco de las intervenciones, a través de un conjunto de prácticas que se dirigen a remitir al actor a sí mismo, a suscitar en sí mismo un trabajo sobre sí, esfuerzos de reconquista de sí, de ocuparse de sí.

En suma, el objetivo de la "creación de empleo" dependería, de esta forma, de un cambio de mentalidad y de cultura que alcanza el corazón de los procesos subjetivos. Por tanto, si algo queda de manifiesto es que más allá del tipo de emprendimiento que pueda crearse (desde una startup innovadora hasta los casos de empleo autónomo de baja calidad), la noción de empleo autónomo que acompaña a la de emprendimiento da muestra de un mecanismo en el cual no solo la creación de empleo sino la participación de los sujetos en distintas esferas sociales depende de las formas en que los individuos pongan en juego sus competencias. Esto nos lleva desde el ámbito del trabajo como empleo al ámbito del trabajo como práctica sobre sí mismo y a partir de sí mismo que los individuos deben poner en acción.

El emprendedor es, en este sentido, un sujeto "empresario de sí mismo" (Foucault, 2007). No alguien que tiene una empresa, sino que gestiona su propia vida como una empresa, como alguien que está todo el tiempo, desde su propia relación consigo mismo y en su relación con los otros, concibiendo, gestionando, organizando su vida como una empresa, y que es responsable tanto de sus éxitos como de sus fracasos. "Europa necesita más emprendedores" condensa entonces la llamada, si no a una "nueva" subjetividad, puesto que su emergencia data de al menos tres décadas, a generar las condiciones de la ampliación de su alcance y desarrollo.

\section{Conclusiones}

Hemos mostrado que el diseño de las medidas de emprendimiento supone un instrumento que orienta la creación de empleo hacia el trabajo autónomo y las microempresas, en línea con algunas de las medidas de activación para el empleo. Por ello, el diseño de una política que propone sostener el crecimiento económico y la creación de empleo a través del emprendimiento, al tiempo que conduce el emprendimien- 
to hacia el empleo autónomo, supone una importante contradicción: al inducir la creación de "emprendimientos" con pocas perspectivas de crecimiento, las políticas parecen fomentar aquello mismo que dicen combatir.

Debe subrayarse que nuestro enfoque no trata de negar o de desconocer que la crisis del empleo típico y las transformaciones del modo de producción requieran plantear otras formas de empleo y de seguridad social. Intentamos, en todo caso, señalar el direccionamiento de esa crisis hacia formas de empleo más mercantilizadas, que hacen un uso abusivo del estatuto de trabajador autónomo (CESE, 2013), y hacia una cultura y una subjetividad moldeada a partir de la lógica empresarial que rompe con las lógicas solidaristas.

Es decir, al colocar al individuo como núcleo de las fuentes de creación de empleo y crecimiento, el discurso europeo señala que es necesario "liberarlo" de los lazos que lo conducirían a la pasividad, a la dependencia y que le impedirían desplegar todo su potencial, su imaginación y su creatividad al servicio de su empleabilidad. Todo ello en un mercado de trabajo que demanda flexibilidad, por lo que esta liberación se traduce en un llamamiento a desplazar la lógica del derecho social y del derecho del trabajo, por considerarlos obstáculos para el despegue de una economía moderna y competitiva, hacia un Estado Social Activo y una sociedad emprendedora.

Las estrategias discursivas que construyen la dicotomía entre el empleo asalariado, signado por una semántica de lo "negativo", y el modelo del emprendedor, valorado positivamente y señalado como una "necesidad", se conjugan con una llamada a la "liberación" del espíritu emprendedor, traducida como la ampliación de la lógica de mercado en detrimento de los mecanismos protectores del trabajo. Esto supone una lógica en la cual algunos individuos mejor posicionados socialmente podrán capitalizar sus competencias y generar emprendimientos exitosos, mientras que otros se verán destinados a "emprender" como un intento de permanecer en el mercado de trabajo de forma precaria.

En este punto, el problema sigue siendo aquel señalado hace ya más de tres lustros por el Informe Supiot (1999): se trata de pensar nuevas formas de ciudadanía y protecciones más "allá del empleo". Es decir, si parece evidente que las fronteras del trabajo asalariado se disuelven, no se trata de acelerar su ruina, sino de buscar formas en las que los modos de empleo a lo largo de la vida de una persona no repercutan en su protección social. Un llamado generalizado hacia el emprendimiento, entendido como empleo autónomo, no parece ser la forma de hacer frente a los nuevos riesgos sociales.

Pero las protecciones sociales son solo una parte del problema, cuya contracara es la marcada tendencia a poner las fuerzas subjetivas al servicio del mercado, bajo el amparo de la figura del espíritu emprendedor. La pretensión universal que hemos destacado en algunos pasajes de estas políticas debe ser problematizada, señalando la arbitrariedad de un discurso que generaliza esta figura. Por ello, hemos intentado contribuir a las reflexiones sobre los efectos subjetivos de los discursos sociales dominantes. La idea de un "sujeto emprendedor" que impulsan las instituciones europeas, caracterizado por la movilidad y la capacidad de adaptación, encaja con las demandas de un mercado de trabajo flexible promovido por las políticas neoliberales. Además, el sujeto emprendedor fomentado por el discurso europeo se encuentra desligado de categorías colectivas. Ese pliegue del individuo sobre sí mismo, responsable de sus éxitos y fracasos, conlleva un intento de neutralizar la conflictividad inherente al mundo del trabajo. En este punto, creemos que debe debatirse pública- 
mente la posibilidad y las formas de "reenviar" al trabajo hacia formas de organización y resistencias colectivas.

\section{Referencias bibliográficas}

Alameda Castillo, M. T. (2016). Emprendimiento y Trabajo Autónomo. Su realidad como mecanismo de incorporación y de permanencia en el mercado de trabajo. Madrid: Ediciones Cinca.

Alonso, L. E. (2007). La Crisis de la ciudadanía laboral. Rubí: Anthropos.

Alonso, L. E. (2011). “¿Gobierno o gestión? El Estado remercantilizador y la crisis de lo social". Encrucijadas: Revista Crítica de Ciencias Sociales, 1: 7-12.

Amigot Leache, P., y Martinez, L. (2013). "Gestión por competencias, modelo empresarial y sus efectos subjetivos. Una mirada desde la psicología social crítica". Universitas Psychologica, 12 (4): 1073-1084.

Audretsch, D. (2007). The Entrepreneurial society. Oxford: Oxford University Press.

Audretsch, D. (2009). "Capital emprendedor y crecimiento económico". Investigaciones Regionales, 15 (2): 27-45.

Ballester Pastor, I. (2016). Trabajo y protección social del autónomo. Un estudio sobre su precariedad. Barcelona: Atelier.

Blanchflower, D. (2004). "Self-Employment: More may not be better". NBER Working Paper Series, 2004.02: 1-30.

BOE (2013). Ley 14/2013, de 27 de septiembre, de apoyo a los emprendedores y su internacionalización. Boletín Oficial del Estado de España.

BOE (2015). Real Decreto Legislativo 3/2015, de 23 de octubre, por el que se aprueba el texto refundido de la Ley de Empleo. Boletín Oficial del Estado Español.

Bourdieu, P. (1985). ¿Qué significa hablar? Economía de los intercambios lingüisticos. Madrid: Akal.

Briales Canseco, Á. (2017). "Emprendeudores fracasados: individualización neoliberal en los discursos sobre el desempleo". Recerca, 20: 79-104.

Bureau, M.-C., y Corsani, A. (2014). «Du désir d'autonomie à l'indépendance». La Nouvelle Revue Du Travail, 5.

Casas Baamonde, M. E. (2013). "El Derecho del Trabajo y el empleo asalariado en los márgenes: de nuevo el emprendimiento y el autoempleo". Relaciones Laborales: Revista Crítica de Teoría Y Práctica, 11: 137-164.

CESE (2013). Comité Económico y Social Europeo. Dictamen sobre el "Uso abusivo del estatuto de trabajador autónomo" (Dictamen de iniciativa). Bruselas. 2013/C 161/03.

CIS (2012). "Actitudes de la juventud en España hacia el emprendimiento. Distribuciones marginales". Madrid: Centro de Investigaciones Sociológicas.

Comisión Europea (2003). "Libro Verde. El espíritu empresarial en Europa". Bruselas: Oficina de Publicaciones de la Comisión Europea.

Comisión Europea (2006). "Aplicar el programa comunitario de Lisboa: Fomentar la mentalidad empresarial mediante la educación y la formación”. Bruselas: Oficina de Publicaciones de la Comisión Europea.

Comisión Europea (2007a). "Competencias clave para el aprendizaje permanente. Un marco europeo". Bruselas: Oficina de Publicaciones de la Comisión Europea. 
Comisión Europea (2007b). "Modernizar la protección social en aras de una mayor justicia social y una cohesión económica reforzada: promover la inclusión activa de las personas más alejadas del mercado laboral". Bruselas COM(2007) 620 final. Oficina de Publicaciones de la Comisión Europea.

Comisión Europea (2009). “El Espíritu Empresarial en la Educación y la Formación Profesionales". Bruselas: Oficina de Publicaciones de la Comisión Europea.

Comisión Europea (2013a). "Cómo crear mentalidades y capacidades emprendedoras en la UE”. Bruselas: Oficina de Publicaciones de la Comisión Europea.

Comisión Europea (2013b). "Plan de acción sobre emprendimiento 2020. Relanzar el espíritu de empresa en Europa”. Bruselas: Oficina de Publicaciones de la Comisión Europea.

Congregado, E., Golpe, A., y Carmona, M. (2010). "Is it a good policy to promote self-employment for job creation? Evidence from Spain". Journal of Policy Modeling, 32 (6), 828-842.

Crespo, E., Prieto, C., y Serrano, A. (2009). Trabajo, subjetividad y ciudadania: paradojas del empleo en una sociedad en transformación. Madrid: Universidad Complutense: CISS.

Crespo Suárez, E., y Serrano Pascual, A. (2013). "Las paradojas de las políticas de empleo europeas: de la justicia a la terapia”. Universitas Psychologica, 12 (4): 1111-1124.

de Castro, J., Justo, R., y Maydeu Olivares, A. (2008). La naturaleza del proceso emprendedor en españa en el contexto internacional. Bilbao: Fundación BBVA.

Desrosières, A. (2014). Prouver et gouverner. Une analyse politique des statistiques publiques. Paris: La Découverte.

Ehrenberg, A. (2010). La société du malaise. Paris: Odile Jacob.

European Commission (2006). Entrepreneurship Education in Europe: Fostering Entrepreneurial Mindsets through Education and Learning. Oslo: Office for Official Publications of the European Communities.

European Commission (2007). Promoting entrepreneurship on TV and in other audio-visual media. Brussels: Office for Official Publications of the European Communities.

European Commission (2012). Flash Eurobarometer 354. Entrepreneurship in the EU and Beyond. Brussels: Office for Official Publications of the European Communities.

Eurostat (2012). Entrepreneurship determinants: culture and capabilities. Luxembourg: Publications Office of the European Union.

Eurydice (2015). La educación para el emprendimiento en el sistema educativo español. Año 2015. Madrid: Ministerio de Educación, Cultura y Deporte de España. Secretaría General Técnica.

Fernández García, A. (2016). “El fomento del autoempleo y del emprendimiento juvenil”. En J. Moreno i Gené y A. M. Romero Burillo (Eds.), Empleo juvenil: entre el desempleo y la precariedad (pp. 643-697). Valencia: Tirant lo Blanch.

Fernández Rodríguez, C. J., y Serrano Pascual, A. (2014). El paradigma de la flexiguridad en las políticas de empleo españolas: un análisis cualitativo. Madrid: Centro de Investigaciones Sociológicas.

Foucault, M. (2007). Nacimiento de la biopolítica. Curso en el Collège de France. (M. et al Senellart, Ed.), Curso en el Collège de France (1978-1979). Buenos Aires: Fondo de Cultura Económica.

García Jiménez, M. (2008). Autoempleo : trabajo asociado y trabajo autónomo. Madrid: Tecnos.

GEM (2016). “Global Entrepreneurship Monitor: informe GEM España”. Santander: Centro Internacional Santander Emprendimiento. 
Grilo, I., y Thurik, R. (2004). “Determinants of Entrepreneurship in Europe”. Rotterdam: Erasmus Research Institute of Management (ERIM). Report Series.

Guillén, A., y Álvarez, S. (2004). “The EU's Impact on the Spanish Welfare State: The Role of Cognitive Europeanization". Journal of European Social Policy, 14 (3): 285-299.

Kenny, K., y Scriver, S. (2012). "Dangerously empty? Hegemony and the construction of the Irish entrepreneur”. Organization, 19 (5): 615-633.

Laval, C., y Dardot, P. (2013). La nueva razón del mundo. Ensayo sobre la sociedad neoliberal. Barcelona: Gedisa.

Luján Alcáraz, J., y Ferrando García, F. (2016). Trabajo Autónomo y fomento del emprendimiento: mitos y realidades. Albacete: Editorial Bomarzo.

Martínez Sordoni, L. (2017). El discurso europeo sobre el emprendimiento. Políticas públicas, trabajo y subjetividad en el marco de la gobernanza económica europea. Amigot Leache, P. e Íñiguez Rueda, L. (dirs.) Departamento de Trabajo Social. Universidad Pública de Navarra, Pamplona.

MESS (2013). "Estrategia de Emprendimiento y Empleo Joven 2013-2016”. Madrid: Ministerio de Empleo y Seguridad Social.

Millán, J., Congregado, E., y Román, C. (2012). “Determinants of self-employment survival in Europe". Small Business Economics, 38 (2), 231-258.

Molina Navarrete, C. (2013). "La dimensión socio-laboral del pretendido -¿o pretencioso?nuevo "estatuto promocional del emprendedor." Estudios financieros. Revista de Contabilidad y Tributación: comentarios, casos prácticos, 369: 177-256.

Nightingale, P., y Coad, A. (2013). "Muppets and gazelles: Political and methodological biases in entrepreneurship research". Industrial and Corporate Change, 23 (1): 113-143.

Parker, S. (2004). The Economics of Self-Employment and Entrepreneurship. Cambridge, UK: Cambridge University Press.

Parker, S. (2009). The Economics of Entrepreneurship. Cambridge, UK: Cambridge University Press.

Pasquier, R. (2005). ““'Cognitive Europeanization” and the territorial effects of multilevel policy transfer: Local development in French and Spanish regions". Regional y Federal Studies, 15 (3): 295-310.

Pérez Vila, M. C. D. (2007). La figura empresarial en el pensamiento economico. Madrid: Unión Editorial, D.L.

Prieto Rodríguez, C. (2013). "Las políticas de empleo en el marco de la metamorfosis de la norma social del trabajo". Clivatge. Estudis i testimonis sobre el conflicte i el canvi socials, 2 (1): 117-129.

Rodríguez-Piñero, M., Casas, M. E., y Valdés Dal-Ré, F. (2013). "La huída del derecho del trabajo hacia el "emprendimiento", las reformas de la Reforma Laboral de 2012 y otras reformas: la L11/2013 y el RDL 11/2013”. Relaciones Laborales: Revista Crítica de Teoría Y Práctica, 10: 1-29.

Román, C., Congregado, E., y Millán, J. (2013). “Start-up incentives: Entrepreneurship policy or active labour market programme?" Journal of Business Venturing, 28 (1): 151-175.

Salais, R. (2010). «Usages et mésusages de l'argument statistique : le pilotage des politiques publiques par la performance». Revue Française des Affaires Sociales, 1-2: 129-147.

Santos Ortega, J. A. (2014). "La política en manos de los empresarios: el imparable ascenso de la ideología del emprendedor". Papeles de Relaciones Ecosociales y Cambio Global, 127: 29-43.

Schumpeter, J. (1942). Capitalism, Socialism and Democracy. London: Routledge. 
Shane, S. (2009). "Why encouraging more people to become entrepreneurs is bad public policy”. Small Business Economics, 33 (2): 141-149.

Suárez Corujo, B. (2017). "Autoempleo (y emprendimiento) juvenil: ¿ahuyentar a los jóvenes de los derechos y garantías laborales?" Cuadernos de Relaciones Laborales, 35 (1): $151-164$.

Supiot, A. (1997). "Informe francés". En A. Marzal (Ed.), Crisis del estado de bienestar y derecho social (pp. 19-27). Barcelona: ESADE. José M. Bosch.

Supiot, A. (1999). Trabajo y empleo: transformaciones del trabajo y futuro del derecho del trabajo : informe para la Comisión Europea. Valencia: Tirant lo Blanch.

Valdaliso Gago, J. M., y López, S. (2007). Historia económica de la empresa. Barcelona: Crítica.

Valdés Alonso, A. (2000). "El trabajo autónomo en España: Evolución, concepto y regulación”. Revista del Ministerio de Trabajo e Inmigración, 26 (6): 13-44.

Vrancken, D., y Macquet, C. (2012). «Du travail sur soi au gouvernement de soi». Informations Sociales, 169 (1): 76-79.

Zangaro, M. (2011). Subjetividad y trabajo: una lectura foucaultiana del management. Buenos Aires: Herramienta. 